\title{
Laser ablation and spectroscopy of copper in liquid and solid ${ }^{4} \mathrm{He}$
}

\author{
P. Moroshkin, ${ }^{*}$ V. Lebedev, and A. Weis \\ Département de Physique, Université de Fribourg, Chemin du Musée 3, CH-1700 Fribourg, Switzerland
}

\begin{abstract}
We present an experimental study of the laser-induced fluorescence spectra of $\mathrm{Cu}$ atoms in bulk liquid and solid helium matrices as well as in the dense plasma created by the laser ablation of copper in liquid helium. We observe transitions of the valence electron and of inner-shell electrons. The former produce structureless line shapes, a large broadening, and a blueshift. The latter practically are not shifted with respect to the free atom and possess a substructure consisting of a zero-phonon line and phonon wings. We suggest a qualitative interpretation of the observed spectra based on the atomic bubble model that takes the lifting of the $\left|M_{J}\right|$ degeneracy due to the bubble deformation and matrix-phonon interactions into account.
\end{abstract}

\section{INTRODUCTION}

Liquid and solid ${ }^{4} \mathrm{He}$ matrices have been used since the early 1990s for matrix-isolation spectroscopy of different metal atoms (work reviewed in Ref. [1]). The most extensive experimental and theoretical studies were carried out with alkali-metal ( $\mathrm{Rb}, \mathrm{Cs})$ and alkali-earth $(\mathrm{Ba})$ elements. The ground states of these elements have spherically symmetric $S$ electronic orbitals, and the structure of the trapping site formed around these atoms is well described by the socalled atomic bubble model [1]. The observed absorption and fluorescence spectra are due to the excitation of the valence electron. The interaction with the surrounding helium atoms strongly perturbs the dopant's valence-electron orbitals, resulting in the shift of the positions of the observed spectral lines toward higher energies and a large broadening. The electronic structure of the transition-metal atoms, such as the coinage-metals $\mathrm{Cu}, \mathrm{Ag}$, and $\mathrm{Au}$, is more complex than the one of the alkali metals. In addition to a single valence $s$ electron, they have ten $(n-1) d$ electrons in a closed shell, giving them a $n S_{1 / 2}$ ground state. Electronic transitions involving the $d$ electrons lie in the visible and near-infrared ranges and make the absorption and fluorescence spectra of coinage metals much richer than those of the alkali metals. The spectroscopy of $\mathrm{Cu}, \mathrm{Ag}$, and $\mathrm{Au}$ atoms embedded in superfluid helium was reported in Ref. [2] where fluorescence emitted by highly excited atomic states formed by the recombination of positive ions and free electrons in superfluid He was observed. Optical excitation spectra of the valence electron were demonstrated for Ag in superfluid helium in Ref. [3].

In a recent paper [4], we reported preliminary results of a laser spectroscopic study of copper and gold atoms in liquid and solid helium. We have shown that the valenceelectron transitions of coinage-metal atoms in condensed helium are strongly broadened and are blueshifted, similar to the well-studied transitions in alkali-metal atoms. On the other hand, inner-shell transitions involving $d$ electrons give rise to extremely narrow spectral lines. We have attributed this behavior to the fact that, in condensed helium, only the outer $n s^{1}$ electronic shell of the dopant atom is perturbed strongly by the interaction with the surrounding helium bulk, while the

\footnotetext{
*peter.moroshkin@unifr.ch

${ }^{\dagger}$ www.unifr.ch/physics/frap/
}

inner $(n-1) d^{10}$ electrons of $\mathrm{Au}$ and $\mathrm{Cu}$ are screened by the outer shell and only are perturbed weakly. A similar effect was observed earlier in experimental studies [5,6] of inner-shell transitions in the rare-earth elements thulium and europium in liquid and solid He.

In the present paper, we present a detailed spectroscopic and time-resolved study of $\mathrm{Cu}$ atoms in normal fluid, superfluid, and solid ${ }^{4} \mathrm{He}$.

\section{EXPERIMENT}

\section{A. Experimental setup}

The experimental setup is described in detail in Ref. [1]. The measurements were performed in normal fluid, superfluid, and solid ${ }^{4} \mathrm{He}$ in a pressure range of 1-40 bar. The sample was produced in a helium pressure cell immersed in superfluid helium cooled by pumping on the helium bath. Pressurized liquid or solid helium matrices were obtained by condensing and then pressurizing helium gas from an external reservoir.

The solid helium matrix was doped with copper atoms and molecules by means of laser ablation with the second $(\lambda=$ $532 \mathrm{~nm})$ or third $(\lambda=355 \mathrm{~nm})$ harmonic of a pulsed Nd:YAG laser focused (from above) onto a solid copper target mounted at the bottom of the cell. The heat deposited by the laser leads to a local melting of the crystal above the target. The ablated material (mostly clusters and nanoparticles) also absorbs the laser radiation and thereby contributes to the heating and local melting of the crystal. By slowly moving the focusing lens away from the target during ablation, we extend the molten helium region in the vertical direction until it extends along the whole inner height of the cell. When the laser is turned off, the sample resolidifies, and the ablated atoms and clusters become trapped in the matrix.

Once the implantation procedure is completed, the implanted atoms are excited by near-UV radiation, using either the third harmonic of the same pulsed Nd:YAG laser or the cw radiation of a UV light-emitting diode (LED). Fluorescence light from the sample volume is collimated by a lens located in the cryostat and then is analyzed by a grating spectrograph (resolution of $0.1 \mathrm{~nm}$ ) equipped with a CCD camera.

Diffusion of implanted atoms in solid He leads to the formation of metal clusters and, thus, to a decrease of the fluorescence signal. When the Nd:YAG laser is used for the excitation, its mean power is set to $\approx 20 \mathrm{~mW}$, a value 
large enough to ensure the simultaneous efficient dissociation of clusters but sufficiently low to prevent a local melting of the sample. Pulses of the same pulsed Nd:YAG laser also were used for cluster dissociation in the experiment using LED light for the atomic excitation. In order to discriminate the LED-induced fluorescence against the Nd:YAG-induced emission, the data acquisition was delayed with respect to the laser pulse by $0.1-1 \mathrm{~s}$.

For experiments in liquid helium, the ablation laser was operated at full power and a repetition rate of $10 \mathrm{~Hz}$ in order to replace copper atoms that diffuse out of the observation volume. We have obtained the best results by using radiation at $355 \mathrm{~nm}$ focused on the target surface and by collecting the fluorescence of the copper atoms excited by the same laser beam approximately $1 \mathrm{~cm}$ above the target. We also have analyzed fluorescence emitted from the ablation spot where the focused powerful laser radiation significantly heats the sample and produces a gas (plasma) bubble with a high density of highly excited copper atoms. Scattered laser light was suppressed by inserting appropriate interference filters.

\section{B. Fluorescence from the ablation spot}

We first discuss the fluorescence spectrum (Fig. 1) emitted from the ablation spot. It is dominated by a continuous

TABLE I. Electronic transitions of $\mathrm{Cu}$ atoms observed in the laserinduced fluorescence spectrum from the ablation spot. Laser ablation by $355-\mathrm{nm}$ radiation in superfluid helium close to the solidification point $(1.5 \mathrm{~K}, 26 \mathrm{bar})$. The wavelengths of the free $\mathrm{Cu}$ atom $\lambda_{\text {free }}$ are taken from Ref. [7].

\begin{tabular}{|c|c|c|c|}
\hline & Transition & $\lambda_{\text {free }}(\mathrm{nm})$ & $\lambda_{\exp }(\mathrm{nm})$ \\
\hline \multirow{17}{*}{$3 d^{9} 4 s 5 s-3 d^{9} 4 s 4 p$} & ${ }^{4} D_{7 / 2}{ }^{4} P_{5 / 2}$ & 427.5 & 428.1 \\
\hline & ${ }^{4} D_{5 / 2}{ }^{4} P_{3 / 2}$ & 437.8 & 438.5 \\
\hline & ${ }^{4} D_{1 / 2}{ }^{4} F_{3 / 2}$ & 450.9 & 451.5 \\
\hline & ${ }^{4} D_{3 / 2}{ }^{4} F_{5 / 2}$ & 453.9 & 454.6 \\
\hline & ${ }^{4} D_{5 / 2^{-}}{ }^{4} F_{7 / 2}$ & 458.7 & 459.4 \\
\hline & ${ }^{4} D_{7 / 2^{-}}{ }^{4} F_{9 / 2}$ & 465.1 & 465.8 \\
\hline & ${ }^{4} D_{5 / 2}{ }^{4} F_{5 / 2}$ & 467.4 & 468.3 \\
\hline & ${ }^{4} D_{7 / 2}{ }^{4} F_{7 / 2}$ & 470.4 & 471.2 \\
\hline & ${ }^{2} D_{5 / 2}{ }^{2} D_{3 / 2}$ & 476.7 & 477.8 \\
\hline & ${ }^{2} D_{3 / 2}{ }^{4} D_{5 / 2}$ & 479.4 & 480.4 \\
\hline & ${ }^{2} D_{5 / 2}{ }^{2} D_{5 / 2}$ & 486.6 & 487.6 \\
\hline & ${ }^{4} D_{1 / 2^{-}}{ }^{4} D_{3 / 2}$ & 501.7 & 502.7 \\
\hline & ${ }^{4} D_{3 / 2}{ }^{2} F_{5 / 2}$ & 503.4 & 504.3 \\
\hline & ${ }^{2} D_{5 / 2}{ }^{2} F_{7 / 2}$ & 507.6 & 508.4 \\
\hline & ${ }^{4} D_{7 / 2^{-}}{ }^{4} D_{7 / 2}$ & 529.2 & 530.3 \\
\hline & ${ }^{4} D_{7 / 2}{ }^{2} F_{5 / 2}$ & 535.3 & 536.6 \\
\hline & ${ }^{4} D_{5 / 2^{-}}{ }^{4} D_{5 / 2}$ & 539.2 & 540.2 \\
\hline \multirow[t]{2}{*}{$3 d^{10} 4 d-3 d^{10} 4 p$} & ${ }^{2} D_{3 / 2^{-}}{ }^{2} P_{1 / 2}$ & 515.3 & 515.9 \\
\hline & ${ }^{2} D_{3 / 2,5 / 2}{ }^{2} P_{3 / 2}$ & $521.8,522.0$ & 522.4 \\
\hline \multirow{3}{*}{$3 d^{10} 4 p-3 d^{9} 4 s^{2}$} & ${ }^{2} P_{3 / 2}{ }^{2} D_{5 / 2}$ & 510.5 & 511.3 \\
\hline & ${ }^{2} P_{3 / 2}{ }^{2} D_{3 / 2}$ & 570.0 & 570.3 \\
\hline & ${ }^{2} P_{1 / 2}{ }^{2} D_{3 / 2}$ & 578.2 & 578.6 \\
\hline \multirow[t]{2}{*}{$3 d^{10} 5 s-3 d^{10} 4 p$} & ${ }^{2} S_{1 / 2}{ }^{2} P_{1 / 2}$ & 793.3 & 796.2 \\
\hline & ${ }^{2} S_{1 / 2}{ }^{2} P_{1 / 2}$ & 809.3 & 812.3 \\
\hline
\end{tabular}

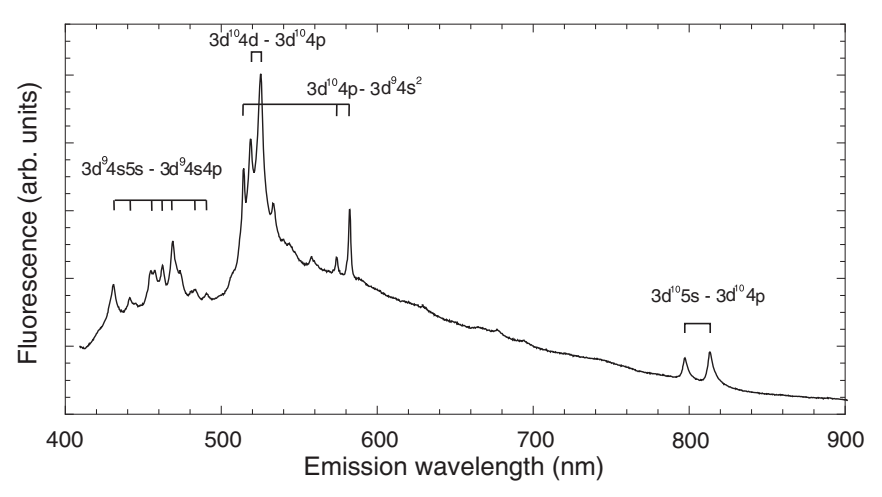

FIG. 1. Fluorescence spectrum from the ablation spot on the solid copper target induced by pulsed 355-nm radiation (third harmonic from the Nd:YAG laser). The target is in superfluid helium close to the solidification point $(1.5 \mathrm{~K}, 26 \mathrm{bar})$.

background extending over 400-800 nm with a maximum at $\approx 520 \mathrm{~nm}$. On top of the background spectrum lies a number of spectral lines of atomic $\mathrm{Cu}$, whose wavelengths are listed in Table I. The resonance doublet of $\mathrm{Cu}$ at 327/325 nm could not be observed because of a strong scattered laser light in that part of the spectrum. The second strongest group of atomic lines represents transitions from the $3 d^{10} 4 p^{2} P_{1 / 2,3 / 2}$ states to the metastable $3 d^{9} 4 s^{2}{ }^{2} D_{3 / 2,5 / 2}$ states. The three lines at 510, 570 , and $578 \mathrm{~nm}$ are clearly visible in Fig. 1 at wavelengths very close to the one in the free $\mathrm{Cu}$ atom. One also can see two pairs of strong emission lines at 516/522 and 796/812 nm. These are transitions from the highly excited $3 d^{10} 5 s^{2} S_{1 / 2}$ and $3 d^{10} 4 d^{2} D_{3 / 2,5 / 2}$ states to the $3 d^{10} 4 p^{2} P_{1 / 2,3 / 2}$ fine-structure doublet (the ${ }^{2} D_{J}$ doublet is not resolved). Both doublets are redshifted slightly with respect to their positions in the free atom (see Table I) and are broadened strongly. The $3 d^{10} 4 d-3 d^{10} 4 p$ doublet is shown on an enlarged scale in Fig. 2(a). The lines have nearly symmetric Lorentzian profiles with a spectral width of $\approx 110 \mathrm{~cm}^{-1}$. At the same time, the $3 d^{10} 4 p-3 d^{9} 4 s^{2}$ lines have a spectral width of only $30 \mathrm{~cm}^{-1}$. All remaining atomic lines shown in Figs. 1 and 2 belong to a large group of transitions between the $3 d^{9} 4 s 5 s$ and the $3 d^{9} 4 s 4 p$ states. Their assignments and wavelengths are given in Table I.

\section{Spectroscopy in bulk condensed helium: Outer-shell transitions}

When laser-induced fluorescence light from the middle of the sample cell ( 1 to $2 \mathrm{~cm}$ above the ablation spot) is observed, the spectrum changes dramatically. The continuous band that dominates the ablation spectrum disappears completely as well as the transitions from the $3 d^{10} 4 p$ and $3 d^{10} 4 d$ states and most of the $3 d^{9} 4 s 5 s-3 d^{9} 4 s 4 p$ lines.

Of all outer-shell transitions of the $\mathrm{Cu}$ atom, the strongest laser-induced fluorescence was observed on the $3 d^{10} 5 s^{2} S_{1 / 2}-3 d^{10} 4 p^{2} P_{1 / 2,3 / 2}$ fine-structure doublet. Two typical spectra obtained in normal fluid helium at low (7-bar) and high (40-bar) helium pressure are shown in Fig. 3. In liquid (both superfluid and normal fluid) helium, these two lines are particularly strong and dominate the spectrum. However, in solid He, their intensity is much lower, and the spectrum is dominated by molecular bands of the $\mathrm{Cu}_{2}$ dimer [8] and by 


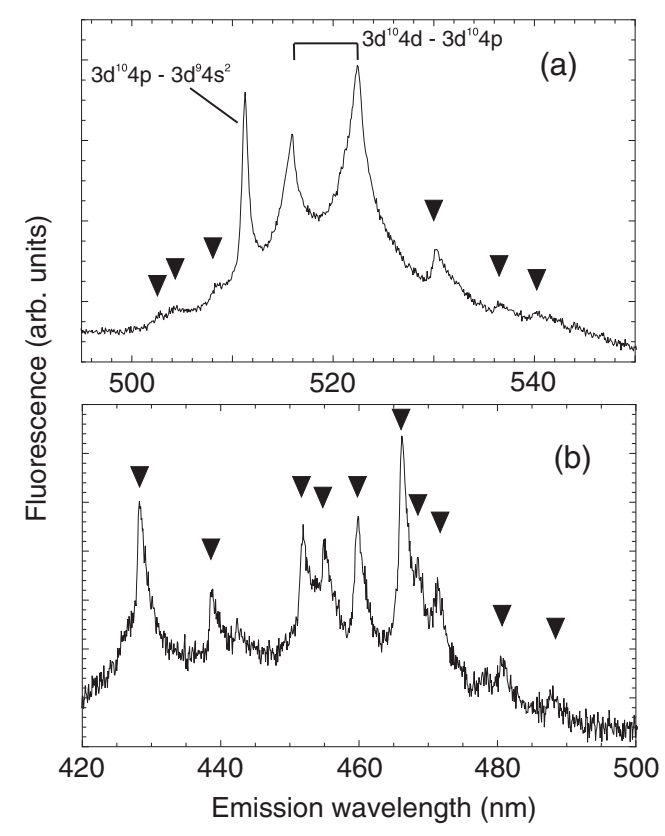

FIG. 2. Closeup of the atomic lines of Fig. 1. The wavelengths of the $3 d^{9} 4 s 5 s-3 d^{9} 4 s 4 p$ transitions (marked by black triangles) are given in Table I.

forbidden inner-shell transitions of $\mathrm{Cu}$ (discussed in Sec. II D below).

In Fig. 3, as one can see, both doublet components have nearly Gaussian line shapes, which differ remarkably from the Lorentzian-like lines observed at the ablation spot. Both lines are broadened strongly and are blueshifted with respect to the transitions in the free $\mathrm{Cu}$ atom. The linewidth and the shift of the red doublet component are plotted in Fig. 4 as a function of the helium pressure for two different temperatures $T=1.5$ and $2.7 \mathrm{~K}$, which correspond to the superfluid and normal fluid phase of helium, respectively. The results obtained in the two phases are nearly identical. The shifts and linewidths increase linearly with pressure in agreement with observations on alkali-metal atoms $[9,10]$, which are well

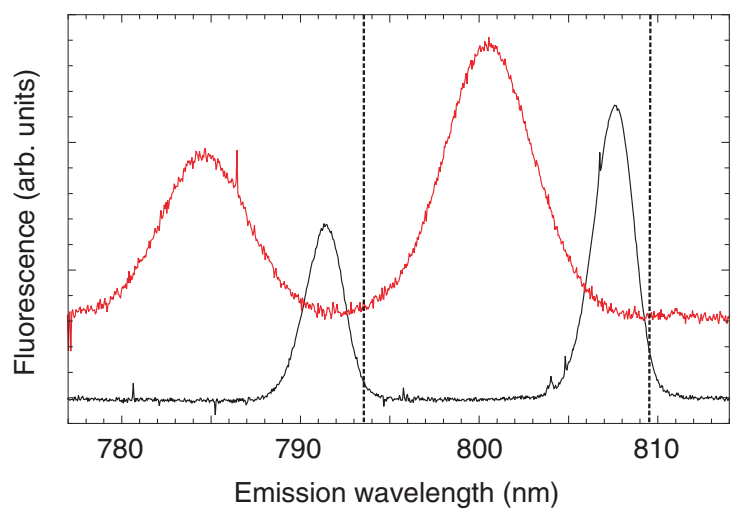

FIG. 3. (Color online) Emission spectra of the $3 d^{10} 5 s^{2} S_{1 / 2}-3 d^{10} 4 p^{2} P_{1 / 2,3 / 2}$ fine-structure doublet of $\mathrm{Cu}$ in liquid He. Lower (black) curve, $T=2.7 \mathrm{~K}$ and $p=7$ bar; upper (red) curve, $T=2.6 \mathrm{~K}$ and $p=40$ bar. Excitation at $355 \mathrm{~nm}$. The vertical dashed lines indicate the doublet wavelengths in the free $\mathrm{Cu}$ atom.

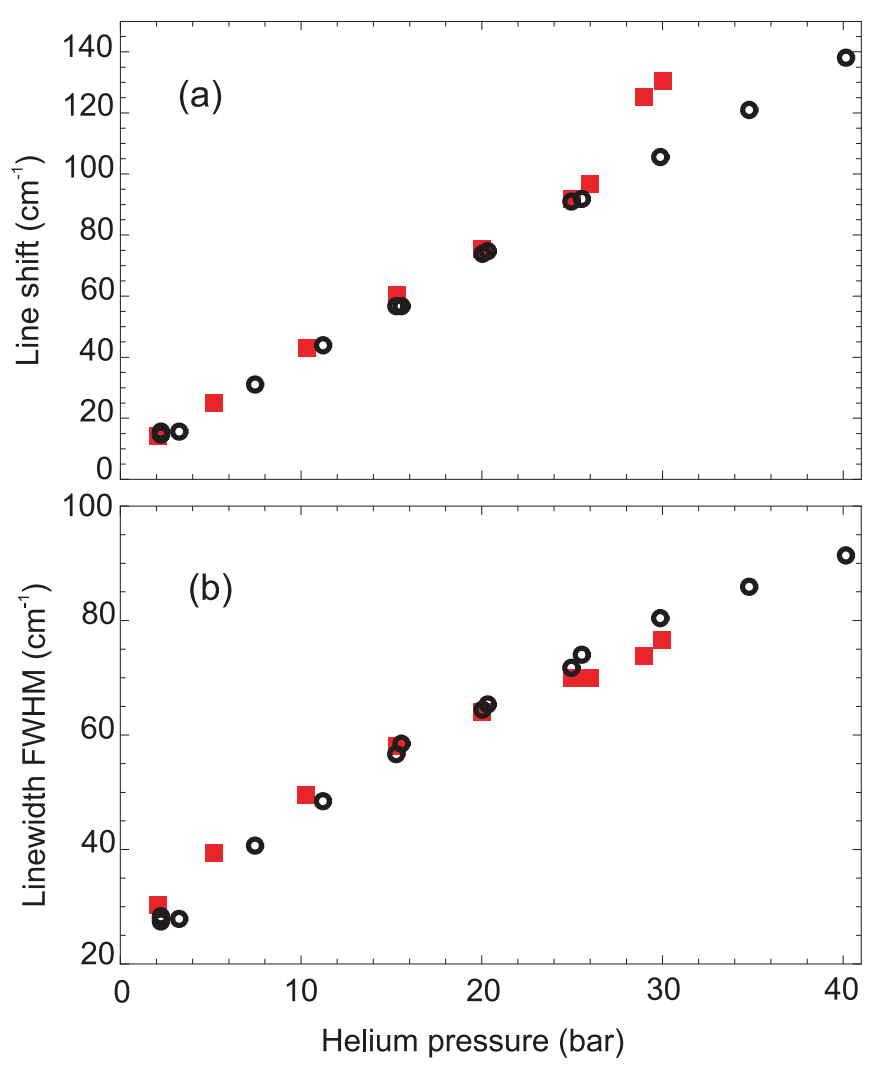

FIG. 4. (Color online) (a) Spectral shift and (b) broadening of the $3 d^{10} 5 s^{2} S_{1 / 2}-3 d^{10} 4 p^{2} P_{3 / 2}$ transition of $\mathrm{Cu}$ as a function of $\mathrm{He}$ pressure. Black circles, $T=2.7 \mathrm{~K}$ (normal fluid $\mathrm{He}$ ); red squares, $T=1.5 \mathrm{~K}$ (superfluid and solid $\mathrm{He}$ ).

described by the atomic bubble model. The two points in Fig. 4 obtained at $1.5 \mathrm{~K}$ above 26 bar correspond to hcp solid He. Unfortunately, in solid $\mathrm{He}$, the $a-X$ band of $\mathrm{Cu}_{2}$ overlaps with the atomic doublet and makes the determination of the shifts and widths less accurate. Nevertheless, one can clearly see a sudden jump in the fluorescence wavelength toward shorter wavelengths at the solidification point. A similar behavior was observed earlier for the resonance lines of $\mathrm{Cs}$ in condensed helium [10].

Besides the $3 d^{10} 5 s^{2} S_{1 / 2}-3 d^{10} 4 p^{2} P_{1 / 2,3 / 2}$ doublet, in solid $\mathrm{He}$, we have observed much weaker fluorescence lines belonging to the $3 d^{9} 4 s 5 s-3 d^{9} 4 s 4 p$ group. These lines in the blue part of the spectrum overlap with the $D-X$ and $B-X$ molecular bands of $\mathrm{Cu}_{2}$ that are particularly strong in solid He. Nonetheless, we clearly can identify three transitions from the ${ }^{4} D_{7 / 2}$ state to the ${ }^{4} P_{5 / 2},{ }^{4} F_{9 / 2}$, and ${ }^{4} F_{7 / 2}$ states at 426.5 , 463.3 , and $\approx 469 \mathrm{~nm}$, respectively.

\section{Spectroscopy in bulk condensed helium: Inner-shell transitions}

Besides the valence-electron transitions discussed above, we have observed laser-induced fluorescence from two excited states created by the promotion of an electron from the inner $3 d$ shell to the outer $4 s$ orbital. Both $3 d^{9} 4 s^{2}{ }^{2} D_{3 / 2,5 / 2}$ states are metastable in the free $\mathrm{Cu}$ atom since the corresponding transitions to the $3 d^{10} 4 s^{2} S_{1 / 2}$ ground state are forbidden. In matrix-isolation studies [11], these transitions were found to 


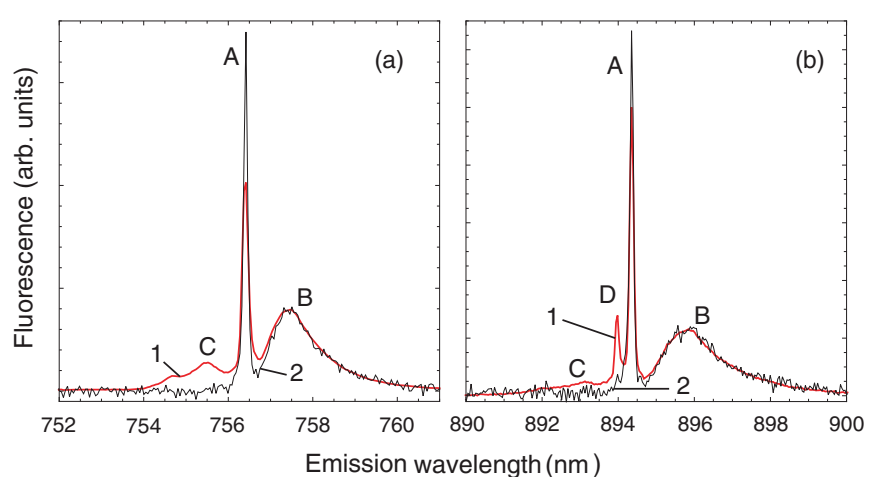

FIG. 5. (Color online) Emission spectra of the (a) $3 d^{9} 4 s^{2}{ }^{2} D_{3 / 2}-3 d^{10} 4 s^{2} S_{1 / 2}$ and (b) $3 d^{9} 4 s^{22} D_{5 / 2}-3 d^{10} 4 s^{2} S_{1 / 2}$ forbidden transitions of $\mathrm{Cu}$ in solid $\mathrm{He}$ at $T=1.5 \mathrm{~K}$ and $p=30$ bar. Curve 1, excitation by pulsed frequency tripled Nd:YAG radiation $(\lambda=355 \mathrm{~nm})$; curve 2 , excitation by $\mathrm{cw}$ LED radiation $(\lambda=307 \mathrm{~nm})$.

be very strong and to dominate the emission spectrum. In condensed helium, we have observed the two lines at $756 \mathrm{~nm}$ $\left({ }^{2} D_{3 / 2^{-}}{ }^{2} S_{1 / 2}\right)$ and $894 \mathrm{~nm}\left({ }^{2} D_{5 / 2^{-}}{ }^{2} S_{1 / 2}\right)$, very close to their wavelengths in the free $\mathrm{Cu}$ atom. In solid $\mathrm{He}$, these lines are much stronger than any of the valence-electron transitions or the molecular bands of $\mathrm{Cu}_{2}$. However, in liquid, and especially in superfluid helium, the two lines become much weaker than all other spectral features. We attribute this effect to the much faster diffusion of $\mathrm{Cu}$ atoms in the liquid compared to the solid matrix. The decay times of these forbidden transitions are very long (see Sec. II E) so that the excited $\mathrm{Cu}$ atoms leave the observation volume before emitting an appreciable amount of fluorescence light.

Typical spectra of the ${ }^{2} D_{3 / 2^{-}}{ }^{2} S_{1 / 2}$ and ${ }^{2} D_{5 / 2^{-}}{ }^{2} S_{1 / 2}$ transitions in a solid He matrix are shown in Figs. 5(a) and 5(b), respectively. In contrast to the nearly Gaussian $5^{2} S_{1 / 2-} 4^{2} P_{1 / 2,3 / 2}$ line shapes, the ${ }^{2} D_{3 / 2,5 / 2^{-}}{ }^{2} S_{1 / 2}$ spectra possess a very pronounced substructure. When pulse excited at $355 \mathrm{~nm}$, the ${ }^{2} D_{3 / 2^{-}}{ }^{2} S_{1 / 2}$ line shape consists of three well-resolved features:

(1) A very narrow central peak [labeled A in Fig. 5(a)] with a spectral width $\gamma_{\text {FWHM }} \lesssim 1.5 \mathrm{~cm}^{-1}$ (spectrograph-limited resolution).

(2) A second peak [labeled B in Fig. 5(a)] that is broader and is redshifted by $\approx 1 \mathrm{~nm}$.

(3) A third feature [labeled C in Fig. 5(a)] that also is broad and is blueshifted with respect to the central peak $A$. This feature has a double structure that is not resolved completely.

The line shape of the ${ }^{2} D_{5 / 2^{-}}{ }^{2} S_{1 / 2}$ transition closely resembles the one of the ${ }^{2} D_{3 / 2}{ }^{2} S_{1 / 2}$ line observed under the same conditions. In addition, it shows a second narrow peak [labeled D in Fig. 5(b)] between peaks $A$ and $C$, which also has a narrow spectrometer resolution-limited width. The shapes and relative shifts of the spectral features $A, B$, and $C$ are very similar to those of the ${ }^{2} D_{3 / 2^{-}}{ }^{2} S_{1 / 2}$ transition.

The shapes and positions of all components of both forbidden lines essentially remain unaffected when the solid He pressure is changed in the range of $28-40$ bar. However, their amplitude ratio depends on the conditions during the sample preparation. In particular, we have noticed that sudden
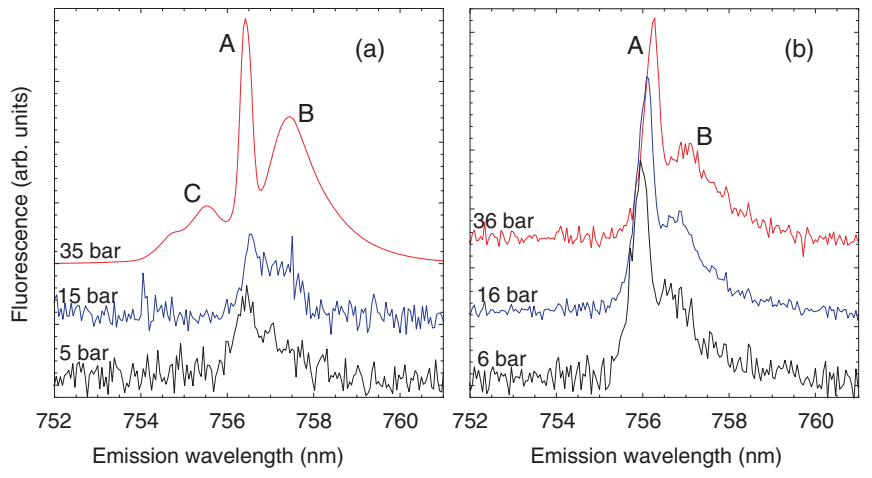

FIG. 6. (Color online) Emission spectra of the $3 d^{9} 4 s^{2}{ }^{2} D_{3 / 2}-3 d^{10} 4 s^{2} S_{1 / 2}$ transition of copper in (a) superfluid and solid $\mathrm{He}$ at $1.5 \mathrm{~K}$ and in (b) normal fluid $\mathrm{He}$ at $2.2 \mathrm{~K}$. Top (red) curves, $p=35$ (36) bar; middle (blue) curves, $p=15$ (16) bar; black (bottom) curves, $p=5$ (6) bar. Excitation at $355 \mathrm{~nm}$.

changes in the helium pressure lead to an amplitude increase of feature $\mathrm{C}$.

In a separate experiment, we have excited $\mathrm{Cu}$ atoms in solid He with low-power cw radiation produced by an LED with a central wavelength of $307 \mathrm{~nm}$ that is nearly resonant with the $3 d^{10} 4 p^{2} P_{1 / 2,3 / 2}-3 d^{10} 4 s^{2} S_{1 / 2}$ lines of $\mathrm{Cu}$. We have observed strong fluorescence from the two forbidden ${ }^{2} D_{3 / 2,5 / 2^{-}}{ }^{2} S_{1 / 2}$ transitions. The corresponding spectra (normalized to the amplitude of peak B) are shown in Fig. 5, where they are compared to the line shapes obtained under Nd:YAG laser excitation. For both transitions, cw excitation increases the relative amplitude of peak $A$, while peaks $C$ and $D$ disappear completely. We have verified that excitation (of the same sample) by the Nd:YAG laser produces all four spectral components.

We have studied the forbidden ${ }^{2} D_{3 / 2,5 / 2^{-}}{ }^{2} S_{1 / 2}$ lines in liquid helium, below and above the $\lambda$ point. In Fig. 6 , we show typical spectra of the ${ }^{2} D_{3 / 2^{-}}{ }^{2} S_{1 / 2}$ transition obtained at $1.5 \mathrm{~K}$ in superfluid and at $2.7 \mathrm{~K}$ in normal fluid helium and compare them to the line shape obtained in solid He. In order to compensate for the low intensity of fluorescence, we had to open the input slit of our spectrograph thereby decreasing its spectral resolution. The apparent broadening of peak $A$ in Fig. 6 is due to the reduced resolution.

In superfluid and normal fluid helium, the two ${ }^{2} D_{3 / 2,5 / 2^{-}}{ }^{2} S_{1 / 2}$ transitions yield only the two peaks $A$ and $B$. The separation between the peaks decreases to $\approx 0.5 \mathrm{~nm}$, and peak $\mathrm{B}$ becomes narrower than in solid helium. We observe no dependence of the line shapes on helium pressure.

\section{E. Dynamics of the inner-shell transitions}

The upper states of the two $3 d^{9} 4 s^{2}-3 d^{10} 4 s$ transitions are metastable. In a gaseous environment, their lifetimes are limited either by atomic collisions or by diffusion to the walls. In solid $\mathrm{He}$, where the atoms are practically immobile, we could observe their fluorescence during $1 \mathrm{~s}$ after the laser pulse. As shown in Figs. 7(a) and 7(c), there is a marked difference between the spectra recorded immediately after the laser pulse and some $100 \mathrm{~ms}$ later. Peak $\mathrm{C}$ dominates the spectrum at short delay times and quickly vanishes, while peaks $A, B$, 

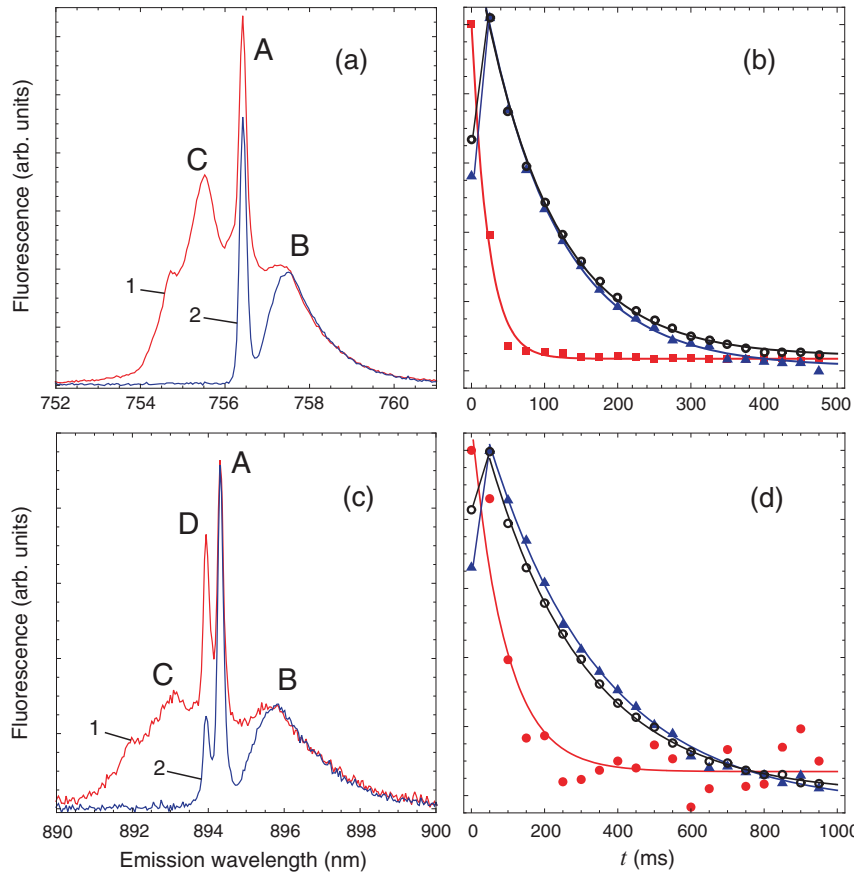

FIG. 7. (Color online) Dynamics of the $3 d^{9} 4 s^{2}-3 d^{10} 4 s$ transitions of copper in solid He following a 5 -ns excitation pulse at $355 \mathrm{~nm}$ (1.5 K, 30 bar). (a) and (b) ${ }^{2} D_{3 / 2^{-}}{ }^{2} S_{1 / 2}$ transition at $756 \mathrm{~nm}$, (c) and (d) ${ }^{2} D_{5 / 2^{-}}{ }^{2} S_{1 / 2}$ transition at $894 \mathrm{~nm}$. (a) and (c) Laser-induced fluorescence spectra at $t=0$ (curve 1) and at $t=100 \mathrm{~ms}$ (curve 2). (b) and (d) Time dependence of the fluorescence intensity at selected wavelengths (triangles, peak A; empty circles, peak B; squares, peak C; filled circles, peak D. Symbols, experimental data; solid lines, exponential fits).

and $D$ persist longer. We have investigated the dynamics of both transitions in solid He by using a short exposure time of $25 \mathrm{~ms}$ for the CCD camera and varying the time delay with respect to the excitation laser pulse. In Figs. 7(b) and 7(d), we show the intensities of different spectral features as a function of the delay time. By fitting the experimental data with exponential decay curves, we have obtained the decay times $\tau_{A}, \tau_{B}, \tau_{C}$, and $\tau_{D}$ of the four peaks. The results are given in Table II.

Although the observed decay times of the two transitions at 756 and $894 \mathrm{~nm}$ are different, their dynamics have several common features that can be clearly seen in Figs. 7(b) and 7(d). In each spectrum, peaks $A$ and $B$ have similar decay times, $\tau_{A} \approx \tau_{B} \approx 100 \mathrm{~ms}$ (transition ${ }^{2} D_{3 / 2^{-}}{ }^{2} S_{1 / 2}$ ) and $\tau_{A} \approx$ $\tau_{B} \approx 300 \mathrm{~ms}$ (transition ${ }^{2} D_{5 / 2^{-}}{ }^{2} S_{1 / 2}$ ). Peak C in both spectra

TABLE II. Measured decay times (ms) of the spectral components $\mathrm{A}, \mathrm{B}, \mathrm{C}$, and $\mathrm{D}$ of the two $3 d^{9} 4 s^{2}-3 d^{10} 4 s$ transitions (Fig. 9) of $\mathrm{Cu}$ in solid $\mathrm{He}$.

\begin{tabular}{lcc}
\hline \hline & ${ }^{2} D_{3 / 2^{-}}{ }^{2} S_{1 / 2}$ & ${ }^{2} D_{5 / 2^{-}}{ }^{2} S_{1 / 2}$ \\
\hline$\tau_{A}$ & $103 \pm 4$ & $303 \pm 4$ \\
$\tau_{B}$ & $96 \pm 3$ & $274 \pm 2$ \\
$\tau_{C}$ & $<20$ & $<40$ \\
$\tau_{D}$ & & $60 \pm 20$ \\
\hline
\end{tabular}

decays much faster and disappears completely within $50 \mathrm{~ms}$ after the laser pulse. Peak D, which is present only in the spectrum of the ${ }^{2} D_{5 / 2^{-}}{ }^{2} S_{1 / 2}$ line (Fig. 5) has a decay time of $60 \mathrm{~ms}$. Although the rising edge of the fluorescence pulse cannot be resolved by our method, in Figs. 7(b) and 7(d), one can see that the intensities of peaks $A$ and $B$ increase during the first 20-50 ms after the laser pulse. The rising times of peaks $C$ and $D$ are much shorter than the minimum exposure time used in the experiment.

\section{DISCUSSION}

\section{A. Excitation mechanism}

First, we address the excitation mechanisms leading to the population of electronic states, observed close to the laserablation spot. As one can see in the energy diagram of the $\mathrm{Cu}$ atom (Fig. 8), none of these states can be populated directly via resonant one- or multiphoton absorption. In fact, all observed states lie above the energy of $28170 \mathrm{~cm}^{-1}$, corresponding to single photons of $355 \mathrm{~nm}$, and the $3 d^{9} 4 s 5 s$ states are even above the two-photon energy $\left(56340 \mathrm{~cm}^{-1}\right)$ and the ionization

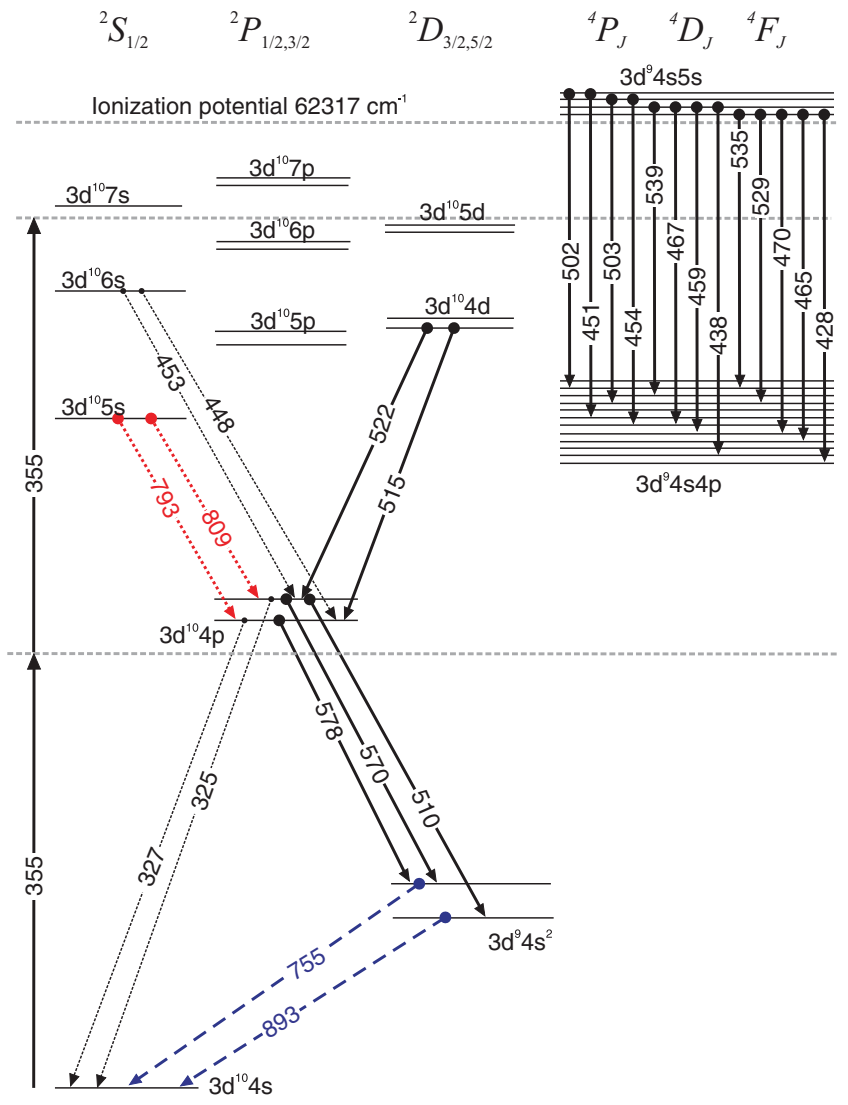

FIG. 8. (Color online) Electronic states of the $\mathrm{Cu}$ atom. Down arrows indicate electronic transitions observed in solid $\mathrm{He}$ (dashed blue lines), bulk liquid $\mathrm{He}$ (dotted red lines), and in the laserinduced plasma at the target surface (solid black lines). Quenched transitions are shown by black dotted lines. Transition wavelengths are given in nanometers. The horizontal dotted lines mark the energies accessible by one- and two-photon excitations at $\lambda=355 \mathrm{~nm}$ (28 170 and $56340 \mathrm{~cm}^{-1}$, respectively) as well as the ionization potential $\left(62317 \mathrm{~cm}^{-1}\right)$. 
threshold of $\mathrm{Cu}$ at $62317 \mathrm{~cm}^{-1}$. We, therefore, suggest that the main population mechanism is via inelastic collisions with free electrons present in the laser-created plasma in the laser focus or via electron-ion recombination.

The situation is quite different in bulk liquid or solid helium far away from the ablation target. The former mechanism most likely does not apply there since all free electrons (if present) rapidly form nanoscopic cavities called electron bubbles [12], which reduce their mobility by orders of magnitude compared to electrons in the gas phase. The mobility of positive ions is also strongly reduced [13] with respect to the gas phase due to the formation of so-called snowball structures (bonding of $\mathrm{He}$ atoms around the positive ion due to electrostrictive attraction). Therefore, recombination processes are expected to be suppressed strongly in condensed helium. The residual weak fluorescence from the $3 d^{9} 4 s 5 s^{4} D_{7 / 2}$ state may be due to recombination.

The main excitation mechanism leading to an efficient population of the $3 d^{10} 5 s$ and $3 d^{9} 4 s^{2}$ states in bulk liquid and solid helium is most likely via quasiresonant two-photon absorption from the ground state toward some highly excited state, followed by a radiationless quenching due to the $\mathrm{Cu}-\mathrm{He}$ interaction. Good candidates for this intermediate state are the $3 d^{10} 5 d$ and $3 d^{10} 6 s$ states since they possess allowed two-photon transitions from the ground state that are redshifted with respect to the UV-laser radiation by 950 and $3490 \mathrm{~cm}^{-1}$, respectively. It is likely that, in condensed helium, these outer-shell transitions are broadened strongly and are shifted toward higher energies, thus, decreasing the detuning with respect to the two-photon energy.

\section{B. Deexcitation cascade}

The laser-excited $\mathrm{Cu}$ atoms decay by a sequential cascade of radiative and radiationless transitions: (1) radiationless quenching toward the $3 d^{10} 5 s$ state, (2) fluorescence at the $3 d^{10} 5 s^{2} S_{1 / 2} \rightarrow 3 d^{10} 4 p^{2} P_{1 / 2,3 / 2}$ fine-structure doublet $(793 / 809 \mathrm{~nm})$, (3) radiationless quenching of the $3 d^{10} 4 p^{2} P_{1 / 2,3 / 2}$ states toward the $3 d^{9} 4 s^{2}{ }^{2} D_{3 / 2,5 / 2}$ metastable states, and finally, (4) fluorescence at the forbidden $3 d^{9} 4 s^{2}{ }^{2} D_{3 / 2,5 / 2} \rightarrow 3 d^{10} 4 s^{2} S_{1 / 2}$ transitions $(755 / 893 \mathrm{~nm})$.

This cascade passes through the $3 d^{10} 4 p^{2} P_{1 / 2,3 / 2}$ states. One, therefore, expects to observe fluorescence at the $3 d^{10} 4 p^{2} P_{1 / 2,3 / 2} \rightarrow 3 d^{10} 4 s^{2} S_{1 / 2}$ transitions, the two strongest lines of the CuI spectrum. We have searched for these two UV-fluorescence lines ( 324.8 and $327.5 \mathrm{~nm}$ in the free atom) but could not detect any corresponding lines within the range of our spectrometer $(\lambda \geqslant 300 \mathrm{~nm})$. At the same time, the three green lines of the $3 d^{10} 4 p^{2} P_{1 / 2,3 / 2} \rightarrow 3 d^{9} 4 s^{22} D_{3 / 2,5 / 2}$ at 510 , 570 , and $578 \mathrm{~nm}$, respectively, also are absent in bulk liquid and solid $\mathrm{He}$, while they are observable nicely in the ablation plasma (see Sec. II B). The excitation with the broadband LED that is nearly resonant with the $3 d^{10} 4 p^{2} P_{1 / 2,3 / 2}-3 d^{10} 4 s^{2} S_{1 / 2}$ lines also produces a strong fluorescence at the forbidden $3 d^{9} 4 s^{2}{ }^{2} D_{3 / 2,5 / 2} \rightarrow 3 d^{10} 4 s^{2} S_{1 / 2}$ lines but no UV nor green fluorescence.

These observations indicate that there is an efficient quenching mechanism for the $3 d^{10} 4 p$ states of copper in liquid and solid helium. At the same time, the two metastable $3 d^{9} 4 s^{2}$ states are populated and emit strong fluorescence at 756 and $894 \mathrm{~nm}$ as described in Sec. II D. We, therefore, conclude that the same process that quenches the $3 d^{10} 4 p^{2} P_{1 / 2,3 / 2}$ states populates the metastable states. As a possible quenching mechanism, we propose the formation of $\mathrm{Cu}^{*} \mathrm{He}_{N}$ exciplexes.

A metal-helium exciplex (quasimolecule) is formed when one or several $\mathrm{He}$ atoms penetrate into the nodal region of the valence-electron's density distribution in the excited metal atom. Dumbbell-shaped $n P$ orbitals of alkali-metal and coinage-metal atoms are particularly well suited for the formation of such complexes. In particular, the $\mathrm{Ag}^{*} \mathrm{He}_{2}$ exciplex was observed in experiments [3] with silver atoms in superfluid $\mathrm{He}$, laser excited to the $4 d^{10} 5 p^{2} P_{3 / 2}$ state. Calculations [15] suggest that $\mathrm{Ag}^{*} \mathrm{He}_{N}$ complexes with $N=15$ can be formed. Similar alkali-helium exciplexes with $1 \leqslant N \leqslant 7$ have also been observed in liquid and solid He [14,16].

Upon exciplex formation, the energy of the $P$ state of the metal atom is lowered because of the van der Waals interaction with the bound He atoms. In the same complex, the energies of the unbound lower states are strongly increased because of the Pauli repulsion between the valence electron of the metal atom and the closed electronic shell of He. The potential curve of the $P$ state, may therefore, cross the one of a lower-lying state. In this case, the probability of a radiationless deexcitation of the complex is strongly enhanced. This mechanism is well known to completely quench the resonance lines of light alkali-metal elements in superfluid and solid helium [17-19]. For copper, we expect a potential curve crossing between the $3 d^{10} 4 p$ and $3 d^{9} 4 s^{2}$ states.

\section{Atomic bubble model}

In order to interpret the experimentally observed spectra, we assume that $\mathrm{Cu}$ atoms in condensed helium reside in nearly spherical atomic bubbles (except for the strongly quenched ${ }^{2} P_{J}$ states). The total energy of the atom + bubble system can be written as $[1,10]$

$$
\begin{aligned}
E_{\mathrm{tot}}= & E_{\mathrm{int}}+p V_{\mathrm{bub}}+\sigma S_{\mathrm{bub}} \\
& +\frac{\hbar^{2}}{8 M_{\mathrm{He}}} \int \frac{[\nabla \rho(R)]^{2}}{\rho(R)} d^{3} R+E_{\text {elastic }},
\end{aligned}
$$

where $p$ is the helium pressure, $\sigma$ is the surface-tension parameter, $V_{\text {bub }}$ and $S_{\text {bub }}$ are the volume and the surface area of the bubble, $\rho(R)$ is the helium density profile at the bubble interface, $E_{\text {int }}$ is the copper-helium interaction energy, and $E_{\text {elastic }}$ is the energy due to the stress created in the solid matrix by the inclusion of the bubble. The same expression, except for the $E_{\text {elastic }}$ term, also applies for liquid helium. The interaction term $E_{\text {int }}$ is mostly due to the repulsive interaction of the copper valence electrons with the closed electronic shells of helium atoms at the bubble interface (Pauli repulsion). This energy rises with a decrease of the bubble radius $R_{\text {bub }}$ when the electronic densities of $\mathrm{Cu}$ and $\mathrm{He}$ start to overlap. The equilibrium bubble size is determined by the balance between $E_{\text {int }}$ and the remaining terms in Eq. (1), which increase with the bubble radius as $R_{\text {bub }}^{2}$ and $R_{\text {bub }}^{3}$, respectively. 
We have evaluated the interaction energy $E_{\text {int }}$ using the theoretical $\mathrm{Cu}-\mathrm{He}$ pair potential from Ref. [20]. The He density around the defect $\rho(\mathbf{R})$ was modeled by a radial trial function,

$\rho\left(R, R_{0}, \alpha\right)= \begin{cases}0, & R<R_{0}, \\ \rho_{0}\left[1-\left\{1+\alpha\left(R-R_{0}\right)\right\} e^{-\alpha\left(R-R_{0}\right)}\right], & R \geqslant R_{0},\end{cases}$

where $\rho_{0}$ is the density of bulk condensed helium.

The parameters $R_{0}$ and $\alpha$ then were adjusted in order to minimize the total atom plus bubble energy (1). For superfluid helium close to the solidification point, we obtain $R_{0}=$ $3.4 \AA$ and $\alpha=3.7 \AA^{-1}$. The corresponding bubble radius $R_{b}$, defined as the center of gravity of the interface profile (2), is equal to $4.0 \AA$. Thus, the copper bubble is significantly smaller than the Cs bubble ( $\left.R_{b} \approx 6 \AA[9,10]\right)$ under similar conditions.

Unfortunately, interatomic $\mathrm{Cu}-\mathrm{He}$ interaction potentials for the excited states of $\mathrm{Cu}$ are not yet available. Therefore, we are unable to calculate the atomic bubble parameters for those states and to predict the corresponding transition energies and line shapes. In the following, we will present a qualitative discussion of the electronic transitions observed in the present experiment.

The electronic transitions in the $\mathrm{Cu}$ atom occur at a fixed bubble configuration. The transition energy of the fluorescence emission is given by

$$
\hbar \omega=\hbar \omega_{0}+\left.\left(E_{\mathrm{int}}^{(e)}-E_{\mathrm{int}}^{(g)}\right)\right|_{R_{\mathrm{bub}}=R_{\mathrm{bub}}^{e}},
$$

where $\omega_{0}$ is the transition frequency in the free atom and the superscripts $e$ and $g$ refer to the electronically excited and ground states, respectively.

First, we consider the fluorescence of the $3 d^{10} 5 s-3 d^{10} 4 p$ fine-structure doublet. The bubble formed around the spherically symmetric $3 d^{10} 5 s$ state is large enough to accommodate the $5 s$ electron orbital. The electronic density of the $3 d^{10} 4 p$ states has a smaller radial distribution and fits easily into this large bubble. Thus, its interaction energy is much smaller than the one of the upper electronic state $E_{\text {int }}^{(e)} \gg E_{\text {int }}^{(g)}$ so that the transition becomes strongly blueshifted. When the He pressure is increased, the bubble radius $R_{\text {bub }}$ decreases, and the excited state is shifted toward higher energies. The blueshift of the transition, thus, increases with helium pressure as shown in Fig. 4. The observed line shapes and the pressure dependence of the line shift and broadening closely reproduce those of the $n s-n p$ transitions of alkali-metal atoms in condensed helium $[1,9,10]$ for which a detailed theoretical analysis is available. Our experiments, thus, suggest that the atomic bubble model also applies to defects formed by transition-metal atoms in liquid and solid helium.

Superfluidity plays no role in the formulation of the atomic bubble model. However, most experiments and theoretical calculations to date were carried out only for dopants in the superfluid phase of helium. Therefore, it is important to compare data obtained in the superfluid and in the normal fluid phases. In Fig. 4, the line shapes and shifts of the outer-shell transitions are the same in both phases. We, therefore, conclude that superfluidity has no significant effect on the atomic bubble configuration.
The interaction between the bubble and the $\mathrm{Cu}$ atom in the metastable $3 d^{9} 4 s^{2}$ state is mostly due to the spherically symmetric $4 s$ electronic shell and, is very similar to the interaction of the $3 d^{10} 4 s$ ground state. The transition frequencies from the two metastable states to the ground state, therefore, are expected to practically be unshifted, i.e., $E_{\text {int }}^{(e)}-E_{\text {int }}^{(g)} \approx 0$. In order to explain the rich substructure of the $3 d^{9} 4 s^{2}-3 d^{10} 4 s$ lines (Fig. 5) discussed below, we consider two mechanisms: lifting of the $\left|M_{J}\right|$ degeneracy in the excited ${ }^{2} D_{J}$ states due to the interaction with the atomic bubble and the interaction of the dopant with phonons.

\section{Interaction of atomic bubbles with phonons}

Electronic transitions in the impurity atom are followed by a shape (size) relaxation of the atomic bubble to a new equilibrium configuration. This expansion or shrinking of the atomic bubble proceeds on a picosecond time scale and creates a localized wave packet of elementary excitations (phonons) in the matrix [4].

Transitions of the valence electron are accompanied by a large displacement of the bubble interface and, therefore, excite a large number of phonons with a broad spectrum. This leads to a strong broadening of the atomic transitions and gives them a structureless Gaussian-like fluorescence spectra.

On the other hand, transitions of the inner-shell electrons leave the bubble size almost unchanged and excite only a small number of phonons. There also is a nonvanishing probability for a transition without excitation of a phonon. Such transitions will appear as a sharp peak-usually called a zero-phonon line (ZPL) - in the fluorescence (absorption) spectrum, very close to the unperturbed transition frequency. Therefore, the fluorescence spectrum will consist of a ZPL and a redshifted broader spectral feature arising from transitions that excite one or several phonons-called the phonon wing (PW). The electronic transition also may be accompanied by absorption of a phonon from the crystal lattice. Such transitions produce a PW that is blueshifted with respect to the ZPL. However, at the temperature of our experiments $(1.5 \mathrm{~K})$, only very few thermal phonons are excited in the crystal, and the blue PW should strongly be suppressed.

\section{E. Lifting of the $\left|M_{J}\right|$ degeneracy in the ${ }^{2} D_{J}$ and ${ }^{2} P_{J}$ states due to the atomic bubble deformations}

Experiments on atomic bubbles formed by alkali-metal ( $\mathrm{Rb}, \mathrm{Cs}$ ) atoms in liquid and solid helium [21,22] have shown that small bubble deformations lead to a removal of the $\left|M_{J}\right|$ degeneracy in the excited $n P_{3 / 2}$ state and to a pronounced splitting of the $n^{2} S_{1 / 2}-n^{2} P_{3 / 2}$ absorption lines. In liquid helium, the deformations arise due to bubble surface vibrations, whereas, in solid hcp matrices, a static deformation appears because of the anisotropic stiffness of the crystal. This anisotropy enters the expression for the bubble energy via $E_{\text {elastic }}$ [the last term in Eq. (1)]. The dominant contribution to the line splitting comes from quadrupolar bubble deformations for which the angular dependence of the bubble radius can be approximated by

$$
R_{\text {bub }}(\theta, \phi)=R_{0}\left[Y_{0}^{(0)}+\beta Y_{0}^{(2)}(\theta, \phi)\right],
$$


where $R_{0}$ is the average (isotropic) bubble radius and $Y_{m}^{(l)}(\theta, \phi)$ are spherical harmonics.

When the bubble deformation is small, $\beta \ll 1$, the interaction Hamiltonian $U_{\text {bub }}$ has the same angular dependence as the bubble radius $R_{\text {bub }}(\theta)$. Evaluation of the matrix elements of $U_{\text {bub }}$ in the basis of the $\left|n, L, S, J, M_{J}\right\rangle$ atomic states shows that the states with an electron angular momentum $J \geqslant 1$ are split into $J+1 / 2$ components with different $\left|M_{J}\right|$.

The splitting of the $3 d^{10} 4 p^{2} P_{3 / 2}$ state into the $\left|M_{J}\right|=1 / 2$ and $3 / 2$ components results in a double structure of the $3 d^{10} 5 s^{2} S_{1 / 2}-d^{10} 4 p^{2} P_{3 / 2}$ fluorescence line (809 nm). However, in our experimental spectra, this line always shows up as a single peak with a symmetric line shape, practically identical to the one of the $3 d^{10} 5 s^{2} S_{1 / 2}-3 d^{10} 4 p^{2} P_{1 / 2}$ line at $793 \mathrm{~nm}$ (see Fig. 3). We, therefore, conclude that the splitting is negligible compared to the linewidth, i.e., $<10 \mathrm{~cm}^{-1}$.

The ${ }^{2} D_{3 / 2}$ state has two substates with $\left|M_{J}\right|=3 / 2$ and $1 / 2$, and the ${ }^{2} D_{5 / 2}$ state is split into three components with $\left|M_{J}\right|=5 / 2,3 / 2$, and $1 / 2$, in order of increasing energy. The selection rule $\Delta M=0, \pm 1$ implies that only the $\left|M_{J}\right|=1 / 2$ and $3 / 2$ substates have transitions to the ground state. Thus, each line will be split into two components with relative intensities determined by the populations of the corresponding $M_{J}$ states, which are populated by radiationless transitions from the $3 d^{10} 4 p$ states.

\section{F. Structure of the inner-shell transitions}

The observed structure of the fluorescence spectra of the $3 d^{9} 4 s^{2}-3 d^{10} 4 s$ transitions cannot be explained by the coupling to phonons nor by the bubble deformation alone. As discussed in Sec. III D, the former mechanism would produce a single narrow ZPL structure and one (or two) broad PW features. The quadrupolar bubble deformation would split each line in two (Sec. III E). We, therefore, suggest that both mechanisms are involved.

In order to compare the line shapes of the two forbidden transitions, in Fig. 9, we plot them against the detuning in energy units. Zero detuning is defined as the position of peak A.

Each of the two spectra in Fig. 9 is normalized to the amplitude of peak B. As one can see from Fig. 9, peaks B in the two spectra have identical shapes and detunings with respect to the central peak $A$. The same holds for peaks $C$, only that the relative intensities of the peaks are different.

We interpret peaks $A$ and $B$ of both transitions as a ZPL and a PW, both originating from the lowest $M_{J}$ component of the excited state. This assignment is based on the fact that (i) both spectral features are present under $\mathrm{cw}$ or pulsed excitation in the liquid as well as in the solid phase; (ii) the two peaks have the same decay times under all experimental conditions. The shape of the PW (peak B) is determined by the properties of the elementary excitations in the matrix. Therefore, it is identical for both transitions and strongly depends on the type of the matrix (liquid or solid). Recently, we have shown [23] that a simple hydrodynamic model may explain the PW shape and its spectral width.

The decay time of the $A+B$ structure in the spectrum of the ${ }^{2} D_{5 / 2}{ }^{2} S_{1 / 2}$ line at $894 \mathrm{~nm}$ is about three times longer than the decay time of the corresponding structure in the ${ }^{2} D_{3 / 2}{ }^{2} S_{1 / 2}$ spectrum at $756 \mathrm{~nm}$. According to our model (Sec. IIIE),

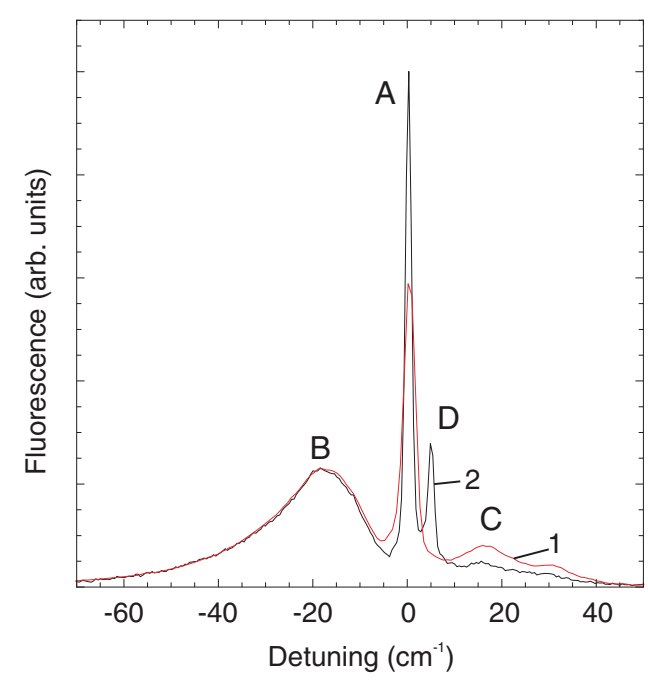

FIG. 9. (Color online) Emission spectra of the $3 d^{9} 4 s^{2}-3 d^{10} 4 s$ transitions of copper in solid He at $T=1.5 \mathrm{~K}$ and $p=30$ bar. Curve 1 (red), ${ }^{2} D_{3 / 2^{-}}{ }^{2} S_{1 / 2}$ transition (756 nm); curve 2 (black), ${ }^{2} D_{5 / 2^{-}}{ }^{2} S_{1 / 2}$ transition $(894 \mathrm{~nm})$. Excitation by the Nd:YAG laser at $355 \mathrm{~nm}$.

the static deformation of the atomic bubble in the hcp crystal should split the ${ }^{2} D_{5 / 2}$ state in such a way that the $\left|M_{J}\right|=5 / 2$ sublevel lies below the $\left|M_{J}\right|=3 / 2,1 / 2$ sublevels. Thus, the transition to the ground state is forbidden by the selection rules for $\Delta M_{J}$ and $\Delta L$. We suggest that the former selection rule is lifted partially by the interaction with the phonons and results in the threefold increase of the decay times $\tau_{A}, \tau_{B}$ compared to the ${ }^{2} D_{3 / 2^{-}}{ }^{2} S_{1 / 2}$ transition.

Peak $D$ is only present in the spectrum of the ${ }^{2} D_{5 / 2}{ }^{2} S_{1 / 2}$ line and is found only in solid $\mathrm{He}$. We assign it to the $\left|M_{J}\right|=3 / 2$ component of the ${ }^{2} D_{5 / 22^{-}} S_{1 / 2}$ transition. In a statically deformed bubble, the energy of the $\left|M_{J}\right|=3 / 2$ states lies above the energy of the $\left|M_{J}\right|=5 / 2$ components, thus, producing a blueshift of $\approx 5 \mathrm{~cm}^{-1}$ with respect to peak $A$. The small spectral width of peak D suggests that it is a ZPL. The associated PW overlaps with the much stronger peaks $A$ and $B$ and, therefore, cannot be observed. The decay time $\tau_{D}$ is much shorter than $\tau_{A}$ and $\tau_{B}$ of the same transition since (i) this transition is allowed by the $\Delta M_{J}=0, \pm 1$ selection rule and (ii) the states $\left|M_{J}\right|=3 / 2$ can decay nonradiatively toward the lower-lying $\left|M_{J}\right|=5 / 2$ states.

Peak $C$ is present in the spectra of both transitions but can only be observed in solid He under pulsed laser excitation. Its blueshift with respect to the other components suggests an assignment to a $\left|M_{J}\right|=1 / 2$ component, which has the largest energy in the statically deformed bubble in the hop phase. This spectral component decays faster than peaks $A$ and $B$, which can be explained by the presence of two decay channels: a radiative channel toward the ground state and a radiationless channel toward the lower-lying $M_{J}$ components of the excited state.

Assuming that component $\mathrm{C}$ is due to a splitting of the $M_{J}= \pm 1 / 2$ components of the excited ${ }^{2} D_{J}$ state, one would expect a spectral shape similar to those of the other components: a sharp ZPL, similar to peaks A and D, and a broader PW, similar to feature B, which both overlap with the other peaks. The observed line shape is quite broad 
with no signature of a ZPL. We, therefore, suggest another interpretation of feature $\mathrm{C}$. This emission may originate from a strong distortion of the atomic bubble by adjacent crystalline defects. These defects may appear due to the local thermal shock experienced by the doped part of the sample from the absorption of the strong UV-laser pulse. Note that $\mathrm{cw}$ excitation produces no emission $\mathrm{C}$. The observed decay time of this emission is associated with the relaxation of the atomic bubble to its equilibrium configuration that has a lower energy.

As discussed in Sec. III C, theoretical $\mathrm{Cu}-\mathrm{He}$ interaction potentials for the excited states of the $\mathrm{Cu}$ atom are not available. The strong ZPLs observed in the spectra of the $3 d^{9} 4 s^{2}-3 d^{10} 4 s$ transitions suggest that the trapping sites of the $3 d^{9} 4 s^{2}$ metastable states are very similar to those of the ground state. The latter can be modeled as a spherical atomic bubble with a radius $R_{b}=4.0 \AA$. The hcp He crystals studied in our experiment have lattice parameters $a \approx 3.7$ and $c \approx 6 \AA$ [24]. A sphere with a radius of $4 \AA$ can be inserted into such a lattice in the space formed by four adjacent vacancies. Thus, it is possible that a four-vacancy center gives a better description of the trapping site in solid He than the spherical atomic bubble. We can speculate that the slow components of the fluorescence spectra (peaks A and B) are emitted from these four-vacancy trapping sites in a perfect hcp lattice, whereas, the fast component (peak C) comes from bubblelike trapping sites in a more disordered solid. In this case, the decay of the fast and the rise of the slow components reflect the dynamics of the recrystallization of the helium matrix following the thermal shock produced by the laser pulse.

\section{SUMMARY}

To summarize, we have carried out a detailed spectroscopic study of copper atoms in liquid and solid helium matrices. The valence-electron transitions in copper behave very similarly to the resonance lines of alkali metals, displaying structureless line shapes with a large broadening and a large blueshift. On the other hand, transitions of inner-shell electrons practically are not shifted and possess a substructure consisting of a ZPL and PWs. We have suggested interpretations of the observed spectra based on the atomic bubble model that takes the lifting of the $M_{J}$ degeneracy due to bubble deformations and interactions with the matrix phonons into account.

\section{ACKNOWLEDGMENTS}

This work was supported by Grant No. 200020-119786 of the Schweizerischer Nationalfonds. P.M. acknowledges financial support from the Light\&Matter (LiMat) project.
[1] P. Moroshkin, A. Hofer, and A. Weis, Phys. Rep. 469, 1 (2008).

[2] B. Tabbert, M. Beau, H. Günther, W. Häußler, C. Hönninger, K. Meyer, B. Plagemann, and G. zu Putlitz, Z. Phys. B 97, 425 (1995).

[3] J. L. Persson, Q. Hui, Z. J. Jakubek, M. Nakamura, and M. Takami, Phys. Rev. Lett. 76, 1501 (1996).

[4] P. Moroshkin, V. Lebedev, and A. Weis, J. Low Temp. Phys. 162, 710 (2010).

[5] K. Ishikawa, A. Hatakeyama, K. Gosyono-o, S. Wada, Y. Takahashi, and T. Yabuzaki, Phys. Rev. B 56, 780 (1997).

[6] Q. Hui and M. Takami, J. Low Temp. Phys. 119, 393 (2000).

[7] A. G. Shenstone, Philos. Trans. R. Soc. London, Ser. A 241, 297 (1948).

[8] V. Lebedev, P. Moroshkin, J. P. Toennies, and A. Weis, J. Chem. Phys. 133, 154508 (2010).

[9] T. Kinoshita, K. Fukuda, Y. Takahashi, and T. Yabuzaki, Phys. Rev. A 52, 2707 (1995).

[10] A. Hofer, P. Moroshkin, S. Ulzega, D. Nettels, R. Müller-Siebert, and A. Weis, Phys. Rev. A 76, 022502 (2007).

[11] G. A. Ozin, S. A. Mitchell, and J. Garcia-Prieto, J. Phys. Chem. 86, 473 (1982).

[12] J. Jortner, N. R. Kestner, S. A. Rice, and M. H. Cohen, J. Chem. Phys. 43, 2614 (1965).
[13] B. Brody, Phys. Rev. B 11, 170 (1975).

[14] P. Moroshkin, A. Hofer, D. Nettels, S. Ulzega, and A. Weis, J. Chem. Phys. 124, 024511 (2006).

[15] M. Mella, M. C. Colombo, and G. Morosi, J. Chem. Phys. 117, 9695 (2002).

[16] K. Hirano, K. Enomoto, M. Kumakura, Y. Takahashi, and T. Yabuzaki, Phys. Rev. A 68, 012722 (2003).

[17] J. Dupont-Roc, Z. Phys. B 98, 383 (1995).

[18] S. Kanorsky, A. Weis, M. Arndt, R. Dziewior, and T. Hänsch, Z. Phys. B 98, 371 (1995).

[19] T. Kinoshita, K. Fukuda, T. Matsuura, and T. Yabuzaki, Phys. Rev. A 53, 4054 (1996).

[20] F. Cargnoni, T. Kus, M. Mella, and R. J. Bartlett, J. Chem. Phys. 129, 204307 (2008).

[21] T. Kinoshita, K. Fukuda, and T. Yabuzaki, Phys. Rev. B 54, 6600 (1996).

[22] V. Lebedev, P. Moroshkin, and A. Weis, J. Phys. Chem. A 115, 7169 (2011).

[23] P. Moroshkin, V. Lebedev, and A. Weis, Europhys. Lett. 96, 26002 (2011).

[24] V. J. Minkiewicz, T. A. Kitchens, F. P. Lipschultz, R. Nathans, and G. Shirane, Phys. Rev. 174, 267 (1968). 